\title{
A study of kinetic molecular exchange processes in the medium frequency range by surface SHG on an oscillating bubble
}

\author{
Jonas Örtegren, Klaus-Dieter Wantke *, Hubert Motschmann, Helmuth Möhwald \\ Max-Planck Institute of Colloids and Interfaces, Am Mühlenberg, D-14476 Golm/Potsdam, Germany
}

Received 4 December 2003; accepted 9 June 2004

Available online 22 July 2004

\begin{abstract}
The dilatational properties of fluid surfaces and interfaces have been comprehensively investigated in recent years. For example, an improved oscillating bubble device provided experimental results that allow for critical testing of established surface models, such as the Lucassen/van den Tempel (LvdT) model. The comparison of the LvdT model with the oscillating bubble experiments demonstrates a mismatch between the model parameters. For example, near the CMC or the limit of solubility the calculated parameters of surfactant solutions become unrealistically large. The deviation can be explained by the introduction of more detailed surface models, in particular by the modification of the effective thickness of the surface layer, its internal structure and the molecular exchange processes between these structures. For the verification of such processes an experimental setup was realized which allows for an independent determination of the instantaneous adsorption state at the surface of an oscillating bubble inside a surfactant solution. The setup utilizes the Second Harmonic Generation (SHG) - effect at the air-solution interface generated by the light of a pulsed LASER. The set-up is described in detail, and the results of a first experimental series are presented and discussed in this paper. As system, aqueous solutions of the fluortenside F381 were used.
\end{abstract}

(c) 2004 Elsevier Inc. All rights reserved.

Keywords: Molecular kinetics; Surface dilatational properties; Surface SHG; Oscillating bubble

\section{Introduction}

\subsection{Aims of the experiments}

Various methods for the investigation of equilibrium properties of fluid surfaces and interfaces are known $[1,2]$. In addition, the results of equilibrium experiments can be transformed for the interpretation of slow dynamic processes at interfaces. But there is a lack of reliable methods to study nonequilibrium states that are caused by faster processes. A fast deformation of the interface can lead to such states. There is up till now no detailed knowledge about the molecular exchange at the surface of a surfactant solution during a fast dilatation. All information about the concentration distributions is the result of indirect methods using measure-

\footnotetext{
* Corresponding author. Fax: +49-331-567-9202.

E-mail address: wantke@mpikg-golm.mpg.de (K.-D. Wantke).
}

ments of dynamic surface tension and special models. Some assumptions of these models are not verified by experiments, like the concentration within the sublayer.

Typical deformation experiments apply longitudinal and transversal surface waves or bubble and drop motions $[3,4]$. In particular, methods using oscillating bubbles or drops are suitable to determine dilatational properties of fluid surfaces and interfaces [5-7]. Their behavior can be described by surface dilatational moduli. These frequency dependent curves exhibit various forms, that are caused by different adsorption and molecular exchange processes at the interface, and therefore the chemical composition and the deformation rates have a decisive influence on these processes. Several models that describe the dynamic behavior of fluid surfaces are established. But experiments in the medium frequency range, e.g., with the oscillating bubble method, exhibit discrepancies to the theoretical models [8-11]. The reason is probably the assumptions about the structure of the adsorption layer under dynamic conditions. Therefore, the modi- 
fication of these models requires more detailed information about the time behavior of the structure. For this purpose, optical experiments that are based on a small monitoring time are essential. By use of a pulsed laser as light source, and by measurement of surface second harmonic generation (SHG), the molecular density of a monolayer can be determined with a time resolution much less than $1 \mathrm{~ms}$. For this reason an experiment was realized which allows the monitoring of the adsorption state at an oscillating bubble on the basis of the SHG effect.

\section{Theoretical aspects}

\subsection{The surface model}

Interesting problems of surface rheology can be explained by discussion of the assumptions that lead to the known expression of the surface dilatational modulus. Here we consider only the models describing oscillating bubbles in surfactant solution. After a short time an adsorption state is established at the surface of an oscillating bubble where all dynamic properties are functions of the relative change in surface area $\Delta A / A=(|\Delta A| / A) \exp (i \omega t)$. Therefore, the surface dilatational modulus, which is defined by the equation

$\varepsilon(f, c)=E(f, c) \exp (i \varphi(f, c))=A \frac{\Delta \gamma^{\prime}}{\Delta A}$,

obtains with

$\gamma^{\prime}=\gamma\left(\Gamma_{0}+\Delta \Gamma(\Delta A)\right)+\kappa(1 / A)(d(\Delta A) / d t)$

the form

$\varepsilon(f, c)=\frac{\Delta \gamma}{\Delta \ln \Gamma} \frac{\Delta \ln \Gamma}{\Delta \ln A}+i \omega \kappa$.

Here $\gamma$ describes the compositional term of the surface tension, $\Gamma=\Gamma_{0}\left(c_{\mathrm{s}}^{0}\right)+\Delta \Gamma\left(\Delta c_{\mathrm{s}}(\Delta A)\right)$ the surface concentration, and $c_{\mathrm{s}}^{0}$ the mean sublayer concentration. The related dynamic expressions are $\Delta \gamma(\Delta A), \Delta \Gamma(\Delta A)$, and $\Delta c_{\mathrm{s}}(\Delta A)$. A more detailed explanation is given later. The first term in Eqs. (2) and (3) is only a function of the surface concentration $\Gamma$ and therefore, some authors have introduced the name compositional contribution to the surface tension. $\kappa$ represents the intrinsic surface dilatational viscosity. It is introduced for formal reasons to characterize the influence of dissipative losses within the surface layer on the surface tension. Experimental results request this term [8-14]. Here we consider only the compositional effects because the solution used shows no influence of an intrinsic surface dilatational viscosity $(\kappa=0)$. Then, Eq. (1) leads to the LvdT modulus if the adsorption is diffusion-controlled. It means that the isotherm equation $\gamma=\gamma\left(\Gamma\left(c_{\mathrm{s}}(t)\right)\right)$ of the surface tension is permanently valid. The LvdT modulus reads [15-17]

$\varepsilon(f, c)=E(f, c) \exp (i \varphi(f, c))=\varepsilon_{\mathrm{g}} \frac{1+\zeta+i \zeta}{1+2 \zeta+2 \zeta^{2}}$, with $\zeta=\sqrt{\frac{\omega_{\mathrm{g}}}{2 \omega}}$.

The parameters

$\varepsilon_{\mathrm{g}}\left(c_{\mathrm{s}}\right)=-d \gamma / d \ln \Gamma \quad$ and $\quad \omega_{\mathrm{g}}\left(c_{\mathrm{s}}\right)=D\left(d c_{\mathrm{s}} / d \Gamma\right)^{2}$

are functions of the sublayer concentration $c_{\mathrm{S}}$ and follow from derivations of the surface tension isotherm equation if $\Gamma$ and $d c_{\mathrm{S}} / d \Gamma$ are determined by the Gibbs adsorption equation. Measured dilatational moduli using the oscillating bubble method demonstrate that Eqs. (4) and (5), in principle, correctly describes the mechanical behavior of surfaces of many surfactant solutions. However, there is a large difference between the parameters $\varepsilon_{\mathrm{g}}\left(c_{\mathrm{s}}\right), \omega_{\mathrm{g}}\left(c_{\mathrm{S}}\right)$ calculated from the surface tension isotherm, and the fit results $\varepsilon_{\mathrm{m}}\left(c_{\mathrm{s}}\right), \omega_{\mathrm{m}}\left(c_{\mathrm{s}}\right)$ of our experimental curves in particular near the CMC or the limit of solubility. In all cases Eq. (4) can be very well fitted to these experimental results, but, in the low concentration range, the experimental Gibbs elasticity, $\varepsilon_{\mathrm{m}}\left(c_{\mathrm{s}}\right)$, is larger than $\varepsilon_{\mathrm{g}}\left(c_{\mathrm{s}}\right)$ whereas for higher concentration near the CMC or the solution limit, $\varepsilon_{\mathrm{m}}\left(c_{\mathrm{s}}\right)$ and $\omega_{\mathrm{m}}\left(c_{\mathrm{s}}\right)$ have too small values. These effects are very often verified by surface rheological measurements with the oscillating bubble method $[9,11]$. The method allows the determination of the modulus up to a magnitude of $150 \mathrm{mN} / \mathrm{m}$ in the frequency range $1 \leqslant f \leqslant 500 \mathrm{~Hz}$. Therefore, the critical concentrations and frequencies can be tested. This is a great advantage of the method. However, the comparison with calculated values includes a principle problem due to the involved second derivation of the measured surface tension isotherm curves. It is known from the numerical mathematics that each derivation of a fit curve increases the error and therefore, the result of the second derivation may be illdefined. However, near the CMC or the solubility limit the calculated parameters become unrealistically great, regardless of the approximation model and at least in this range the difference between $\varepsilon_{\mathrm{m}}\left(c_{\mathrm{s}}\right)$ and $\varepsilon_{\mathrm{g}}\left(c_{\mathrm{s}}\right)$, respectively $\omega_{\mathrm{m}}\left(c_{\mathrm{s}}\right)$ and $\omega_{\mathrm{g}}\left(c_{\mathrm{s}}\right)$, must be considered as verified. The comparison of experimental and calculated parameters was discussed in our previous articles in detail $[9,11]$. It leads to the conclusion that for the explanation of the results in critical concentration ranges additional, independent experiments are required. SHG experiments provide such information.

For a detailed interpretation of the exchange processes the thickness of the effective surface layer and the surface concentration $\Gamma$ must be defined. Most authors use the Gibbs adsorption model with the position of the dividing plane in a real monolayer. Then, the influence of the sublayer is neglected and the surface concentration resulting from the Gibbs equation is interpreted as monolayer concentration $\left(\Gamma=\Gamma_{\mathrm{m}}\right)$. This model should be modified. In Fig. 1 the effective surface layer is split into a topmost monolayer and an adjacent sublayer of thickness $d$. Below this layer the system has pure bulk properties. In an equilibrium state it is reasonable to neglect the influence of the sublayer since its influence cannot be detected by static experiments due to the fixed relations between different defined concentrations in 


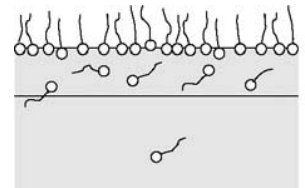

monolayer

sublayer

bulk

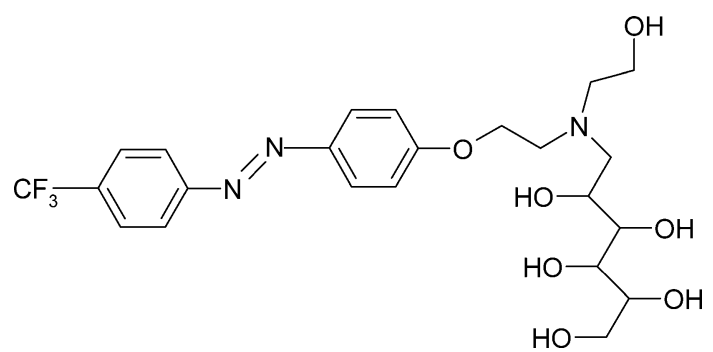

Fig. 1. Upper part: the slightly modified surface model used in this work and in previous work. According to this model, the effective surface consists of a monolayer and a sublayer, and the properties of the surface are partly governed by the exchange dynamics between the two layers. Lower part: the surfactant molecule F381 used in this work.

these cases. However, for the explanation of dynamic experiments such details are important. Therefore, we consider the consequence of the extended effective surface layer on the interpretation of the dynamic experiments. In such a model the surface concentration $\Gamma$ is given by

$\Gamma=\Gamma_{\mathrm{m}}+\Gamma^{*} \quad$ with $\Gamma^{*}=\int_{-d}^{0} c(x) d x$.

The related surface concentration $\Gamma_{\mathrm{w}}$ of the solvent is given by a similar expression. The fictive concentration $\Gamma^{\prime}$ which fulfills the Gibbs adsorption equation [18,19],

$\Gamma^{\prime}=\Gamma-\frac{c}{c+c_{\mathrm{w}}} \Gamma_{\mathrm{w}}=-\frac{1}{R T} \frac{d \gamma}{d \ln c}$,

adopts the value of the monolayer concentration of the surfactant, $\Gamma^{\prime} \cong \Gamma_{\mathrm{m}}$, due to the approximations $\Gamma \cong \Gamma_{\mathrm{m}}+c * d$, $\Gamma_{\mathrm{w}} \cong c_{\mathrm{w}} * d$, and $\Gamma_{\mathrm{w}} * c /\left(c+c_{\mathrm{w}}\right) \cong c * d$ which are valid for a low concentrated solution $\left(c_{\mathrm{W}} \gg c, c_{\mathrm{W}}, c\right.$ average concentration of the solvent $\left(c_{\mathrm{W}}\right)$ and the surfactant $(c)$ in the sublayer). Therefore, in an equilibrium state the value of $\Gamma^{\prime}$ is invariant against a variation of the layer thickness and we cannot conclude that the effective surface area is restricted to a monolayer. According to Eq. (7) the concentration $\Gamma$, used in the parameter definition Eq. (5), cannot be derived from the Gibbs adsorption equation if $\Gamma$ deviates from $\Gamma^{\prime} \cong \Gamma_{\mathrm{m}}$. This modification has no influence on the form of the modulus Eq. (4) but on its parameters. The results of the oscillating bubble experiments hint to the introduction of the proposed modified definition of the effective surface concentration described by $\Gamma$ because the difference between the concentration $\Gamma^{\prime}$ and $\Gamma$ can explain the discrepancy between the calculated and measured parameters of the established LvdT model [11]. The calculation of the parameters on the basis of the isotherm equation remains only correct if $\Gamma^{*}$ is negligible.
For the estimation of this value we consider the law of mass conservation, which describes the molecular exchange between surface layer and bulk phase independently of the model in the following form:

$$
\begin{aligned}
\frac{1}{A} \frac{d n}{d t} & =\frac{\Gamma}{A} \frac{d(A(t))}{d t}+\frac{d \Gamma}{d t}=-\left.D \frac{\partial c}{\partial x}\right|_{x=-d} \\
& =-(1+i) \sqrt{\frac{D \omega}{2}} \frac{d c_{\mathrm{s}}}{d \Gamma} \Delta \Gamma,
\end{aligned}
$$

where $n$ is the number of surfactant molecules within the surface layer and $D$ the bulk diffusion coefficient. In Eq. (8) the solution to the diffusion equation

$$
\begin{aligned}
\Delta c(x, t) & =c(x, t)-c_{0} \\
& =\left|\Delta c_{\mathrm{s}}\right| \exp \left((1+i) k^{\prime} x+i \omega t+i \beta^{*}\right)
\end{aligned}
$$

is used for $x<-d$. Here $c_{0}$ represents the bulk concentration far from the surface, $\beta^{*}$ the phase shift to $\Delta A / A$, $k^{\prime}$ the wave number of the diffusion wave, and $x$ the coordinate perpendicular to the surface. The comparison of Eqs. (6) and (9) shows that the parameter $\Delta \Gamma / \Delta c_{\mathrm{s}}$ depends on $d$. For $d=0$ the expression $\Delta \Gamma / \Delta c_{\mathrm{s}}$ can be approximated by the derivation $d \Gamma_{\mathrm{m}} / d c_{\mathrm{s}}$ of the isotherm equation $\Gamma_{\mathrm{m}}=\Gamma_{\mathrm{m}}\left(c_{\mathrm{s}}\right)$, whereas, we obtain for $d>0$ the unequation $\left(\Delta \Gamma / \Delta c_{\mathrm{s}}\right) \leqslant d \Gamma_{\mathrm{m}} / d c_{\mathrm{s}}$ as demonstrated in the following.

The molecular exchange between bulk and the sublayer is negligible in the high frequency range. Consequently, the number, $n$, of molecules within the surface layer remains constant, which means

$\Delta n \approx 0$ or $\left(\omega_{\mathrm{g}}^{\prime} / 2 \omega\right)^{1 / 2} \Delta \Gamma \approx 0$.

The mark indicates the modification of the parameters due to the modification of $\Gamma$. Therefore, the surface tension isotherm equation cannot be used for the calculation of

$\varepsilon_{\mathrm{g}}^{\prime}\left(c_{\mathrm{s}}\right)=-d \gamma / d \ln \Gamma$ respectively $\omega_{\mathrm{g}}^{\prime}\left(c_{\mathrm{s}}\right)=D\left(d c_{\mathrm{s}} / d \Gamma\right)^{2}$.

With the approximation Eq. (10), Eq. (8) leads to

$\Delta \Gamma / \Gamma=-\Delta A / A$,

which is fulfilled for $\omega_{\mathrm{g}}^{\prime} \ll \omega$ caused by small values of $d c_{\mathrm{S}} / d \Gamma$ also for an equilibrium state at the surface. Then, the surface dilatational modulus becomes constant with a zero phase angle. This can be verified by experiments with the oscillating bubble method where the frequency range is sufficiently high and large magnitudes are measurable. For $d=0$ the parameters $\varepsilon_{\mathrm{g}}^{\prime}$ and $\omega_{\mathrm{g}}^{\prime}$ adopt the theoretical values $\varepsilon_{\mathrm{g}}$ and $\omega_{\mathrm{g}}$ which can be calculated using, in Eq. (5), the surface tension isotherm equations, since $\Gamma$ represents the monolayer concentration $\Gamma_{\mathrm{m}}$ in this case. The problem of the verification of the assumption due to the involved second derivation is discussed above. Here we consider only the higher concentration range where $\varepsilon_{\mathrm{g}}$ exceeds realistic limits and $\varepsilon_{\mathrm{g}}$ must be replaced by $\varepsilon_{\mathrm{g}}^{\prime}$. Then Eq. (8), respectively (12), describes only the molecular exchange at the plane $x=-d$. In the level range of the modulus this exchange is 
negligible, which is however not the case for the exchange between monolayer and sublayer. Nevertheless, the modulus is given by the LvdT model, if the thermodynamic equilibrium between monolayer, sublayer and adjacent topmost layer of the bulk is instantaneously established. For the replacement of $\varepsilon_{\mathrm{g}}$ by $\varepsilon_{\mathrm{g}}^{\prime}$, respectively $\omega_{\mathrm{g}}$ by $\omega_{\mathrm{g}}^{\prime}$, in Eq. (4) the thickness $d$ must be small compared to the wavelength $\lambda$ ( $\sim 1000 \mathrm{~nm}$, for $f<1000 \mathrm{~Hz}$ ) of the diffusion wave and therefore also $d * \Delta c_{\mathrm{s}} \ll \Delta \Gamma$.

Then, the amplitude of the oscillation of the bulk concentration $\Delta c(x, t)$ at $x=-d$ can be estimated using the fit results of the bubble measurements in Eq. (11). That means

$\varepsilon_{\mathrm{g}}^{\prime} \cong \varepsilon_{\mathrm{m}} \quad$ and $\quad \omega_{\mathrm{g}}^{\prime} \cong \omega_{\mathrm{m}}$,

which leads to the formula

$\Delta c_{\mathrm{s}}=\sqrt{\omega_{\mathrm{m}} / D} \Delta \Gamma$.

This interpretation is justified because the parameter $\omega_{\mathrm{m}}$ characterizes the real molecular exchange between surface layer and bulk. The only difference to the standard interpretation of a diffusion-controlled process is the replacement of the monolayer by an extended layer.

Then, the continuity Eq. (8), the solution Eq. (9), and Eq. (14) yield the equation

$$
\begin{aligned}
\frac{\Delta A}{A} & =\left(-\left.\frac{D}{i \omega} \frac{\partial c}{\partial x}\right|_{x=-d}-\Delta \Gamma\right) \frac{1}{\Gamma} \\
& =\left((-1+i) \sqrt{\frac{\omega_{\mathrm{m}}}{2 \omega}}-1\right) \frac{\Delta \Gamma}{\Gamma}
\end{aligned}
$$

for the relation between the relative change in the surface area and the relative change in effective surface concentration for the modified LvdT model. It leads in the high frequency range, defined by a limit frequency $f_{\mathrm{g}}\left(c_{\mathrm{S}}\right)$, to Eq. (12). Therefore, the relative change in the effective surface concentration $\Gamma$ is given by this equation if the surface dilatational modulus reaches a constant level $\varepsilon\left(f, c_{\mathrm{S}}\right)=$ $\varepsilon_{\mathrm{m}}\left(c_{\mathrm{S}}\right)=$ const. with vanishing phase angle in the range $f>f_{\mathrm{g}}\left(c_{\mathrm{s}}\right)$. The unequality $\varepsilon_{\mathrm{m}}\left(c_{\mathrm{s}}\right) \cong \varepsilon_{\mathrm{g}}^{\prime}\left(c_{\mathrm{s}}\right)<\varepsilon_{\mathrm{g}}\left(c_{\mathrm{s}}\right)$ yields the unequality

$$
\Delta \Gamma / \Gamma>\Delta \Gamma_{\mathrm{m}} / \Gamma_{\mathrm{m}}
$$

because of

$\varepsilon_{\mathrm{m}}=-\frac{\Delta \gamma}{\Delta \ln \Gamma_{\mathrm{m}}} \frac{\Gamma}{\Delta \Gamma} \frac{\Delta \Gamma_{\mathrm{m}}}{\Gamma_{\mathrm{m}}} \cong \varepsilon_{\mathrm{g}} \frac{\Gamma}{\Delta \Gamma} \frac{\Delta \Gamma_{\mathrm{m}}}{\Gamma_{\mathrm{m}}}$.

Therefore, the measured dilatational moduli suggest that the effective surface concentration $\Gamma$ near the CMC or the limit of solubility becomes larger than the monolayer concentration $\Gamma_{\mathrm{m}}$. The aim of the SHG experiment was the verification of the inequality (16). A positive result supports strongly the hypothesis of an extended effective surface layer in the case of higher concentrated solutions. Independent of the model we can say that a diffusion-controlled response of the surface tension is detectable by the frequency behavior of the surface dilatational modulus.

\subsection{Second harmonic generation (SHG)}

Second order nonlinear optical processes such as SHG and sum frequency generation can selectively probe interfaces [20-22]. The reason is that second order nonlinear optical processes are not stimulated in centrosymmetric media in the electric dipole approximation. Therefore, the bulk of a liquid does not contribute to the second order nonlinear optical signal. At the surface, however, the inversion symmetry is broken, and generation of, e.g., second harmonics is allowed. The generation of second harmonic light at the interface can be described in terms of the polarization induced at the interface by the incoming light. If the disturbance of the polarization by the incoming light is small, as compared to the binding forces between the nuclei and the electrons, the polarization can be expressed in a power series:

$P_{i}=\varepsilon_{0}\left(\chi_{i j}^{(1)}+\chi_{i j k}^{(2)} E_{k}+\cdots\right) E_{j}$,

where the convention of summation over repeated indices was adopted. $P_{i}$ is the polarization along a Cartesian coordinate axis $i, \varepsilon_{0}$ is the permittivity of free space, $E_{j}$ the electric field of the incoming light polarized along $j, \chi^{(1)}$ the linear susceptibility, and $\chi^{(2)}$ the second-order susceptibility. The subscripts of the susceptibilities denote a particular projection depending on the interacting fields. A second order nonlinear optical material/surface is characterized by having a nonzero $\chi^{(2)}$.

In analogy with the description of the macroscopic polarization, the polarization $p$ of a molecule is described by

$p_{i}=\mu_{i}+\varepsilon_{0}\left(\alpha_{i j}+\beta_{i j k} E_{k}+\cdots\right) E_{j}$,

where $i, j, k$ make up the coordinate system at the molecular level, $\mu_{i}$ is the ground state dipole moment, $\alpha_{i j}$ is the polarizability, and $\beta_{i j k}$ is the second order polarizability. SHG measurements at the air-water interface with a surfactant that has the same SHG-active group as the surfactant used in this work have been reported in the literature [23,24]. The second order polarizability of the chromophore is completely dominated by its $\beta_{z z z}$ component, with $z$ being along the long chromophore axis. Therefore, the other $\beta$ components can be neglected, and the $\beta$ tensor can be treated as a scalar quantity. This simplifies the analysis a great deal. Under certain simplifying assumptions, the oriented gas model provides a relation between the effective second-order susceptibility $\left(\chi_{\text {eff }}^{(2)}\right)$ and $\beta$ according to

$\chi_{\text {eff }}^{(2)} \propto \sum_{\substack{\text { molecules } \\ \text { at surface }}} \beta \propto \Gamma_{\mathrm{m}}\langle\beta\rangle$,

where $\Gamma_{\mathrm{m}}$ is the concentration of $\beta$-active units in the monolayer, and $\langle\beta\rangle$ (here $\langle\beta\rangle=\left\langle\beta_{z z z}\right\rangle$ ) is the spatial and orientational average of the second order polarizability. The reflected or transmitted second harmonic light intensity $\left(I^{2 \omega}\right)$, being generated at an interface, scales to $\chi_{\mathrm{eff}}^{(2)}$ and the incoming light intensity $\left(I^{\omega}\right)$ in the following way:

$I^{2 \omega} \propto\left(\chi_{\mathrm{eff}}^{(2)}\right)^{2}\left(I^{\omega}\right)^{2}$. 
By combination of Eqs. (20) and (21), the relative surfactant concentration of the monolayer, $\Gamma_{\mathrm{m}}$, is obtainable by measurement of the SHG light intensity, $I^{2 \omega}$.

\section{Experimentals}

\subsection{Materials}

Various concentrations of aqueous solutions of the fluortenside F381, depicted in Fig. 1, were studied by tensiometry and SHG. Measurements were done in the static state on the flat air-solution interface, and in the dynamic state in the oscillating bubble configuration. An aqueous solution of F381 was prepared. This fluorchemical-based surfactant was prepared by H. Prescher and purified by chromotography with methyl chloride on silica-gel (Merk). Water was purified with a Milli-Q system. Below we discuss the influence of impurities on the results. The UV-spectra were measured in an Agilent $8453 \mathrm{UV}$-vis spectrophotometer. The equilibrium surface tension $(\gamma(c))$ was measured in a Krüss tensiometer K11.

\subsection{Measurement of the surface dilatational modulus with the oscillating bubble method}

The surface dilatational modulus $\varepsilon(f, c)$ was determined with the oscillating bubble method $[4,8,9]$. Within a closed chamber a small hemispherical bubble is produced at the tip of a capillary. A piezoelectric translator generates a sinusoidal oscillation of the bubble volume and consequently produces changes in surface area and radius. This causes sinusoidal modulations of the pressure in the chamber, which is measured by a pressure transducer at the bottom of the chamber. The chosen bubble diameter $(\sim 0.4 \mathrm{~mm})$ and oscillation amplitude allowed measurement of harmonic oscillations up to approximately $500 \mathrm{~Hz}$. In this range the pressure response remains linear if the relative deformation $|\triangle A / A|$ remains smaller than 0.15 . We apply normally $|\Delta A / A|=0.05$. The oscillating bubble measurements were carried out on 6 concentrations $(200,150,100,60,30$, and $10 \mu \mathrm{M}$ ) of aqueous solutions of the fluorchemical-based surfactant F381. Water and decanoic acid solutions were used for calibration of the measurement.

\subsection{SHG experiments}

The SHG measurements were carried out on five concentrations $(150,60,30,15$, and $10 \mu \mathrm{M})$ of aqueous solutions of the fluortenside F381, depicted in Fig. 1, in two different setups. In both setups the SHG measurements were carried out with the frequency-doubled light at $532 \mathrm{~nm}$ of an active/passive mode-locked Nd:YAG-laser (B.M. Industries YAG 502 DPS 7910DP). The laser pulses were $35 \mathrm{ps,} \mathrm{and} \mathrm{the}$ repetition rate $10 \mathrm{~Hz}$. The light was plane-polarized before the surfactant solution using a Glan-Thomson prism (extinction ratio $10^{-6}$, Type K, Steeg \& Reuter). The plane of polarization was rotated $39^{\circ}$ from vertical polarization, where the SHG apparent molecular tilt angle is independent of the true distribution mean [25]. Spectral purity of the incoming light was assured by use of an IR cut-off filter (BG39, Schott) in conjunction with a narrow-band interference filter (532 BP, LOT-Oriel). The frequency-doubled light generated at the interface was separated from the fundamental light by use of a visible cut-off filter (UG5, Schott) and a narrowband interference filter (266 BP5, LOT-Oriel). The SH light was detected by a photomultiplier tube (R1398, Hamamatsu) with a quantum efficiency of $15 \%$, and a cathode radiant sensitivity of $35 \mathrm{~mA} \mathrm{~W}^{-1}$ at $266 \mathrm{~nm}$. The supply voltage on the photomultiplier tube was ca. $1850 \mathrm{~V}$ (V5D, Seefelder Messtechnik). The detected signal was processed by an oscilloscope (54720D, Hewlett-Packard) and integrated by a computer program.

\subsection{SHG at the flat air-solution interface}

In the first SHG setup, the equilibrium surfactant density was measured on the flat air-solution interface. The surfactant solution was poured into a beaker, and left to stabilize for a few minutes before measuring. The fundamental light was focused by a lens $(F=60 \mathrm{~mm})$, and was incident at the air-solution interface at an angle of incidence of $61^{\circ}$, and partly reflected into the detector. At least 10 measurements were performed on each concentration, where each measurement is the average of the intensity of 100-200 laser-pulses of SH-light reflected at the surface. The measurements lasted approximately $10 \mathrm{~min}$, and no changes in the reflected SHintensity could be detected during this time, indicating that equilibrium prevailed during the measurements.

\subsection{SHG in the oscillating bubble configuration}

In the second SHG setup, SHG measurements were carried out on an oscillating bubble. A detailed description of the setup is given elsewhere [26]. A closed chamber, schematically depicted in Fig. 2, was filled with the surfactant solution. A capillary with inner diameter of ca. $2 \mathrm{~mm}$ was silanized and broken, leaving the capillary walls hydrophobic and the fracture area hydrophilic. The capillary was placed in the closed chamber, according to Fig. 2, and a hemispherical bubble was formed at the end of the capillary. A piezoelectric translator (LVPZT, P830.40, Physik Instrumente) is connected to the chamber, and generates a sinusoidal deformation of the bubble volume and consequently of the surface area and the bubble radius. The voltage over the piezoelectric translator is generated by a computer and amplified (LVPZT-amplifier, model E-501.00, Physik Instrumente) before reaching the translator. The amplitude of the sinusoidal voltage over the piezoelectric translator was $30 \mathrm{~V}$. The frequencies used were 10, 20, 40, and $60 \mathrm{~Hz}$. For frequencies above $60 \mathrm{~Hz}$, the shape of the bubble deviates a 

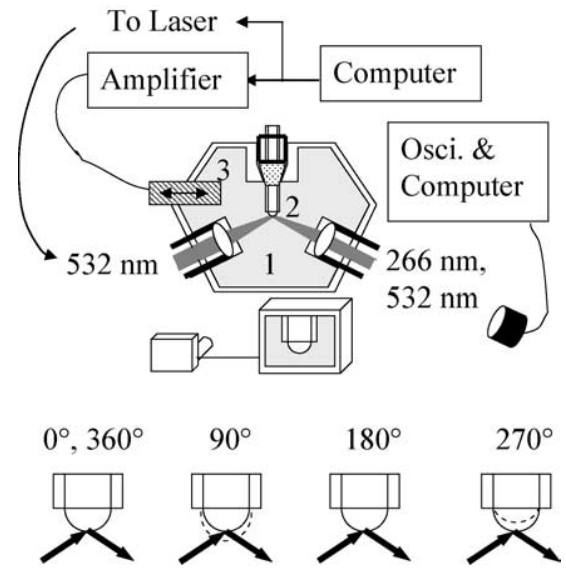

Fig. 2. The chamber with the oscillating bubble used in the nonequilibrium SHG experiments. The fundamental light $(532 \mathrm{~nm})$ is focused by a lens onto the oscillating bubble at an angle where total reflection occurs. The fundamental and generated second harmonic light is collected by a quarz-lens. 1: surfactant solution. 2: capillary. 3: piezoelectric translator. Bottom: definition of the phase angle $\psi$ during bubble oscillation. The dotted curve is the volume of the bubble in equilibrium, and the full curve is the volume of the bubble as it is hit by light.

little from the spherical geometry due to the oscillations. Therefore, the reflected light is spread differently, and the comparison of the results is uncertain.

The computer also triggers the laser, and the trigger signal can be freely chosen during the sinusoidal voltage cycle. The fundamental light was focused by a lens ( $F=16 \mathrm{~mm}$, 063033, Linos Photonics) attached to an adjustable screw, and was incident in the total reflection mode at the bottom of the bubble at an angle of incidence of $68^{\circ}$. The frequencydoubled light generated at the bottom of the bubble was collected by a quarz-lens ( $F=20 \mathrm{~mm}, 063316000$, Linos Photonics) attached to an adjustable screw at the rear end of the chamber (see Fig. 2). At least 10 measurements were performed at each phase angle, where each measurement is the average of the intensity of 100-200 laser-pulses of SH-light reflected at the bubble surface.

The shape and size of the oscillating bubble in the SHG setup was studied by optical means. The image of the bubble was detected by a CCD-camera (EHDkamPro02, S/N: 0053, EHD Imaging $\mathrm{GmbH}$ ) and transferred to a screen. A sharp image of the bubble during oscillation was obtained by using the ps laser pulses as the only light source. According to these observations, the shape of the bubble was hemispherical in the studied frequency range [26].

We define the phase angle $\psi$ of the bubble during oscillation as $90^{\circ}$, and $270^{\circ}$, when the bubble obtains its minimum and maximum volume, respectively. The SHG signal related to one adjustment of $\psi$ was measured independently of the other settings. This allows a small change of the mean volume of the bubble between the measurements. In the case of a phase angle of $\psi=90^{\circ}$, the mean volume of the bubble was made slightly larger than a half-sphere, so that the bubble was a half-sphere as it was hit by light. Analogously, in the case of a phase angle of $\psi=270^{\circ}$, the mean volume of the bubble was made slightly smaller than a half-sphere, again so that the bubble was a half-sphere as it was hit by light. In the case of the phase angles $0^{\circ}$ and $180^{\circ}$ the bubble was a half-sphere both in equilibrium and as it was hit by light. Consequently, light hit the bubble at exactly the same position and same size of the bubble at all $\psi$. The definition of $\psi$ is illustrated in Fig. 2.

\section{Results and discussion}

\subsection{Equilibrium properties measured by $\mathrm{SHG}$ and tensiometry}

In order to test the SHG analysis method, we want to compare the SHG results (yielding $\Gamma_{\mathrm{m}}$ ) with the results from tensiometry (yielding the $\gamma(c)$ adsorption isotherm). This can be done by a direct comparison of both quantities. The surfactant concentration of the monolayer $\Gamma_{\mathrm{m}} \cong \Gamma^{\prime}$ can be retrieved from the $\gamma(c)$ adsorption isotherm through the Gibbs adsorption equation (Eq. (7)). The analysis involves the differentiation of $\gamma(c)$ with respect to $c$. This differentiation is however critically dependent on small fluctuations in the $\gamma(c)$-curve that are unavoidable due to experimental inaccuracy. A more appealing approach in terms of combining numerical mathematics with experimental physics is the integration of $\Gamma_{\mathrm{m}}$ with respect to $c$, described below.

SHG was measured in equilibrium at the flat air-liquid interface of various concentrations of F381, yielding, by use of Eqs. (20) and (21), the $\Gamma_{\mathrm{m}}(c)$ adsorption isotherm depicted in Fig. 3. The surface tension $\gamma_{\mathrm{m}}(c)$ was obtained from Eq. (7) by integration of $\Gamma_{\mathrm{m}}(c)$ with respect to $c$, and by least-square fitting of a proportionality constant to $\gamma(c)$ measured by tensiometry. In Fig. 3 the results are demonstrated as a function of bulk concentration. In comparing $\gamma_{\mathrm{m}}(c)$ and $\gamma(c)$ we must bear in mind that surface SHG detects only the monolayer concentration $\Gamma_{\mathrm{m}}(c)$ [27,28]. We also assumed that the derivation of the measured surface tension $\gamma_{\mathrm{m}}(c)$ yields, according to Eq. (7), the monolayer concentra-

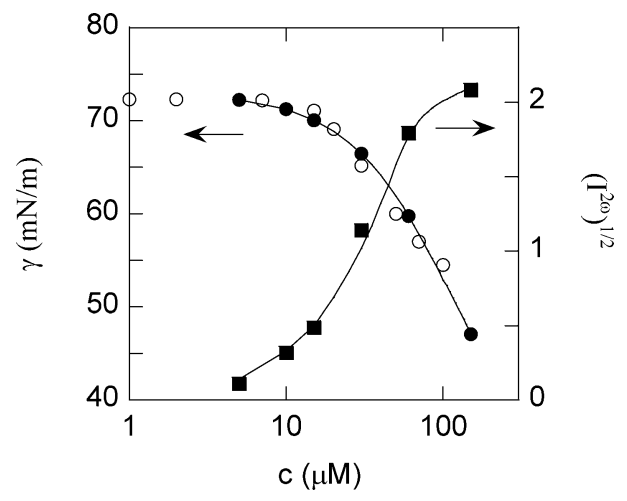

Fig. 3. Comparison of the equilibrium surface tension measured by tensiometry $(\gamma(c), \bigcirc)$, and calculated from the SHG signal $\left(\gamma_{\mathrm{m}}(c), 0\right)$ as a function of bulk concentration of surfactants. The latter was calculated by integration of the adsorption isotherm $\left(\Gamma_{\mathrm{m}}(c)\right.$, 
tion because of $\Gamma^{\prime}(c)=\Gamma_{\mathrm{m}}(c)$. The relatively close overlap between $\gamma(c)$ and $\gamma_{\mathrm{m}}(c)$ indicates that the assumptions are realistic in equilibrium. The small deviations between the two curves may be ascribed to the different measurement techniques. The relatively close overlap is further a motivation for comparative tensiometric studies and SHG studies in the oscillating bubble configuration.

\subsection{Tensiometry in the oscillating bubble configuration}

The surface dilatational modulus $\varepsilon(f, c)$ of various concentrations of F381 in water was measured with the oscillating bubble method. Fig. 4 depicts $\varepsilon(f, c)$ of six concentrations as a function of oscillation frequency. The amount of the surface dilatational modulus is largely constant with frequency in the frequency range $10 \leqslant f \leqslant 500 \mathrm{~Hz}$ for $c \leqslant 150 \mu \mathrm{M}$ concentrations. The phase angle $\varphi$ between the deformation of the bubble and the measured pressure modulation in the chamber is $0 \leqslant \varphi \leqslant 5^{\circ}$ at and above $20 \mathrm{~Hz}$ for $c \leqslant 150 \mu \mathrm{M}$, and increases with lower frequencies. The large phase angles at the lower frequencies are due to bulk diffusion, which depends on the concentration (the phase angle increases more strongly for $f \rightarrow 0$ with higher concentration). The experimental results imply that bulk diffusion is comparatively small already at $10 \mathrm{~Hz}$, and negligible at and above $20 \mathrm{~Hz}$. The constant value of $\varepsilon$ with frequency and the decrease of $\varphi$ down to zero with increasing frequency, are equivalent to a surface that is purely elastic. Such behaviour, including the bulk diffusion at the lower frequencies, can be described by the LvdT modulus (Eq. (4)). In the slightly extended model including a surface consisting of a sublayer and monolayer described in this paper and in previous pa-

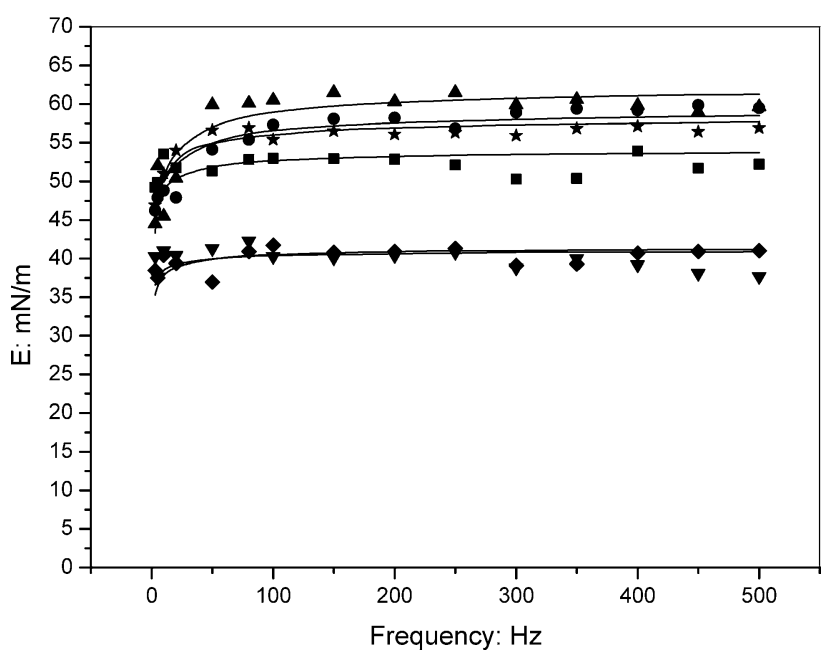

Fig. 4. Measured magnitude $E$ of the surface dilational modulus of aqueous solutions of F381: $200 \mu \mathrm{M}$ : (A) $E$, additional information: experimental phase angle: $\varphi(10 \mathrm{~Hz})=16^{\circ}, \varphi(20 \mathrm{~Hz})=6^{\circ} ; 150 \mu \mathrm{M}:(\bigcirc) E$, $\varphi(10 \mathrm{~Hz})=14^{\circ}, \varphi(20 \mathrm{~Hz})=5^{\circ} ; 100 \mu \mathrm{M}:(\star) E, \varphi(10 \mathrm{~Hz})=8^{\circ}$, $\varphi(20 \mathrm{~Hz})=3^{\circ} ; 60 \mu \mathrm{M}:(\mathbb{a}) E, \varphi(10 \mathrm{~Hz})=9^{\circ}, \varphi(20 \mathrm{~Hz})=1^{\circ} ; 30 \mu \mathrm{M}$ : ( $) E, \varphi(10 \mathrm{~Hz})=9^{\circ}, \varphi(20 \mathrm{~Hz})=3^{\circ} ; 10 \mu \mathrm{M}:(>) E, \varphi(10 \mathrm{~Hz})=9^{\circ}$, $\varphi(20 \mathrm{~Hz})=1^{\circ}$; (-): related theoretical curves (LvdT-model) using the parameters of Table 1 .
Table 1

\begin{tabular}{llllcl}
\hline $\begin{array}{l}C \\
(\mathrm{mMol} / \mathrm{l})\end{array}$ & $\begin{array}{l}\Gamma_{i} \\
\left(10^{10} \mathrm{Mol} \mathrm{cm}^{-2}\right)\end{array}$ & $\begin{array}{l}\varepsilon_{\mathrm{g}} \\
(\mathrm{mN} / \mathrm{m})\end{array}$ & $\begin{array}{l}\varepsilon_{\mathrm{m}} \\
(\mathrm{mN} / \mathrm{m})\end{array}$ & $\begin{array}{l}\omega_{\mathrm{g}} \\
\left(\mathrm{s}^{-1}\right)\end{array}$ & $\begin{array}{l}\omega_{\mathrm{m}} \\
\left(\mathrm{s}^{-1}\right)\end{array}$ \\
\hline 0.010 & 1.0 & 2.5 & 43 & 4.9 & 1.1 \\
0.030 & 3.0 & 8.0 & 44.5 & 6.4 & 1.7 \\
0.060 & 5.1 & 19.7 & 54.5 & 176 & 1.4 \\
0.100 & 6.4 & 69.5 & 59.0 & 243 & 3.3 \\
0.150 & 6.4 & $>500$ & 60.0 & $>1000$ & 4.4 \\
0.200 & 5.9 & $>1000$ & 63.3 & $>1000$ & 6.1 \\
\hline
\end{tabular}

pers [8-11], the experimental results may be explained if the molecular exchange between the sublayer and the monolayer is fast in comparison to the deformation rate. The monolayer, the sublayer and the adjacent topmost layer of the bulk are then in equilibrium during the whole deformation cycle, and the model becomes equal to the LvdT model. Parameters of the model were determined by fitting of the oscillating bubble measurements $\left(\varepsilon_{\mathrm{m}}\left(c_{\mathrm{s}}\right), \omega_{\mathrm{m}}\left(c_{\mathrm{s}}\right)\right)$ and by calculations using the isotherm measurements $\left(\varepsilon_{\mathrm{g}}\left(c_{\mathrm{S}}\right), \omega_{\mathrm{g}}\left(c_{\mathrm{s}}\right)\right)$. For the calculations of the parameters the approximation and differentiation procedures of the Origin-software were applied (Lorentz fit). It was only the aim to demonstrate that also in the case of the F381 solutions, the isotherm equation leads to unacceptable dynamic parameters. For other solutions this was demonstrated in previous papers $[9,11]$. The principle problems of such comparison are discussed above.

We do not look in detail at the influence of impurity on the dynamic effects. This influence on the static surface tension is comprehensively discussed in the literature [29], however, reliable information about the dynamic case is not available. According to our experience, impurities lead to a shift in the surface tension isotherm. However, in the parallel range of these curves their influence on the dilatational modulus is negligible because of the insignificant molecular exchange due to the very low bulk concentration of the impurity. We could verify this behaviour by comparison of surface dilatational moduli of solutions purified according to the recommendation in [29], with products as received in a few cases. The used F381 surfactant is a laboratory product, which was purified by chromotography. Its dynamic properties are similar to the tested solution and we assume that the influence of its impurities on the modulus is also small. In addition, the impurity of the solution cannot be evaluated by the behaviour of the surface tension near the CMC because the F381 solution has a solubility limit of $c=2.3 \times 10^{-4} \mathrm{~m}$ $\left(\gamma\left(2.3 \times 10^{-4} \mathrm{~m}\right)=39.6 \mathrm{mN} / \mathrm{m}\right)$. However, for the comparison of rheological experiments with the SHG experiments it was mainly important that all measurements were done with the same solution during a short time period. In Table 1 the results of the dilatational experiments are demonstrated. It underlines the known fact that the values $\varepsilon_{\mathrm{g}}$ and $\omega_{\mathrm{g}}$ calculated on the basic of isotherm measurements, differ widely from the fit-results of the experiments, $\varepsilon_{\mathrm{m}}$ and $\omega_{\mathrm{m}}$. The totally unrealistic values of the calculations in the higher concentration range are not documented in the table. SHG experiments should help to understand the effect. 


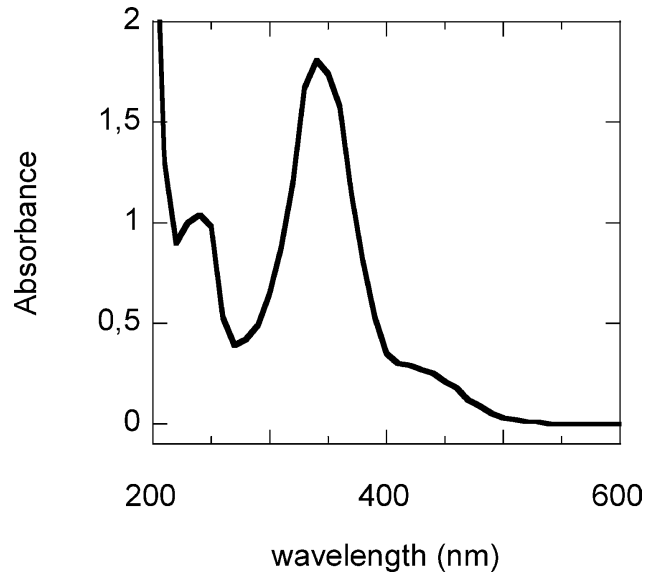

Fig. 5. UV-spectra of $1 \mathrm{~cm}$ of $150 \mu \mathrm{M}$ F381.

\subsection{SHG in the oscillating bubble configuration}

An important prerequisite for the SHG investigation in the oscillating bubble setup described in this paper is demonstrated in Fig. 5, where the UV-spectrum of F381 is shown. The adsorption maximum occurs at about $340 \mathrm{~nm}$, and the solution is transparent at $532 \mathrm{~nm}$, and exhibits a dip in absorption close to $266 \mathrm{~nm}$. Consequently, the resonance wavelength of the system is close to the given wavelengths of light (giving resonance enhancement of SHG), and the absorption from the solution is low. These features make the system well suited for SHG measurements in the oscillating bubble configuration where the fundamental light has a wavelength of $532 \mathrm{~nm}$.

The shape of the bubble was hemispherical and the relative amplitude $(\triangle A / A)$ of the area of the bubble during oscillation was $0.18 \pm 0.04$ as studied by optical means [26]. The value of $\triangle A / A$ was chosen to give a variation in the SHG signal with the phase angle $\psi$ that was indisputably larger than the error of the measurements. In addition, the variation of the square root of the SHG signal is proportional to $\triangle A / A$ what in the hemispherical geometry leads to an approximately linear response of $\Delta \Gamma_{\mathrm{m}} / \Gamma_{\mathrm{m}} \sim \Delta A / A$ as the experiments demonstrate. The presented tensiometric studies on the oscillating bubble show that kinetic exchange processes do not limit the molecular flow at the surface, meaning that an equilibrium state prevails within the surface layer. The measurements further indicate that the diffusive molecular exchange between the bulk and the surface is comparatively small already at $10 \mathrm{~Hz}$. According to the tensiometric studies on the oscillating bubble, we therefore expect that the number of molecules at the surface remains constant at all bubble oscillation frequencies that are available with the present SHG setup (10-60 Hz). As mentioned above, Eq. (8) then leads to the condition $\Delta \Gamma / \Gamma=-\Delta A / A$. Here the thickness of the effective surface layer is not clearly defined, however, it must be small in comparison to the wavelength $\lambda$ of the diffusion wave. Only if the surfactant density of the monolayer fulfils the condi-

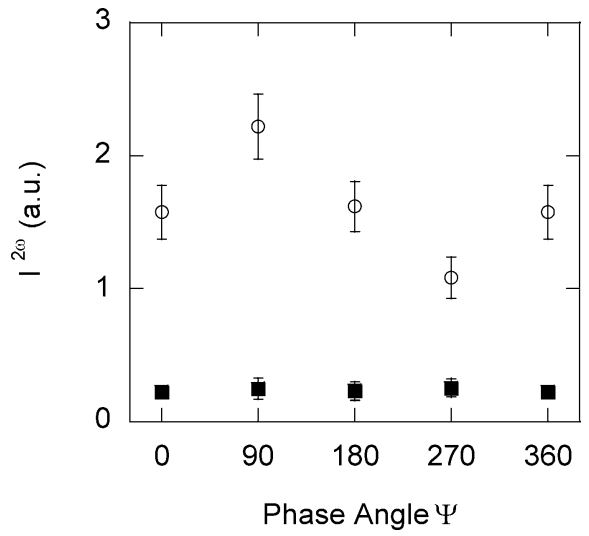

Fig. 6. SH intensity reflected by the oscillating bubble, as a function of phase angle $\psi$. (O) $c=10 \mu \mathrm{M}, f=40 \mathrm{~Hz}$; (ם) water.

tion

$\frac{\Delta \Gamma_{\mathrm{m}}}{\Gamma_{\mathrm{m}}}=-\frac{\Delta A}{A}$

we can assume that the monolayer represents the effective surface layer.

In Fig. 6, one SHG measurement on an oscillating bubble at four phase angles $\psi$ is shown. After calibration against water, the normalised surfactant density of the monolayer as a function of $\psi$ may be calculated by use of Eqs. (20) and (21), giving a $\Delta \Gamma_{\mathrm{m}} / \Gamma_{\mathrm{m}}$ ratio of 0.21 for the measurement shown in Fig. 6. Several measurements were performed for each concentration and oscillation frequency. The ratio $\Delta \Gamma_{\mathrm{m}} / \Gamma_{\mathrm{m}}$ was, on average, about 0.18 for the three lowest concentrations used in this study, and deviated less than $20 \%$ from 0.18 for all individual measurements. Consequently, the ratio $\Delta \Gamma_{\mathrm{m}} / \Gamma_{\mathrm{m}}$ was, within experimental accuracy, equivalent to $-\Delta A / A$, meaning that Eq. (22) holds for the three lowest concentrations. This is realistic for the low concentrated solutions, whereas, near the limit of solubility or the CMC, Eq. (16) indicates that the quotient $\left|\Delta \Gamma_{\mathrm{m}} / \Gamma_{\mathrm{m}}\right|$ becomes smaller than $|\Delta A / A|$ also in the level area of the modulus $\left(\varepsilon(f, c)=\varepsilon_{\mathrm{m}}\right)$ where the molecular exchange with the bulk $(x<-d)$ is negligible. This means that the effective surface layer is extended, which corresponds to Eq. (16), with the unequality $\varepsilon_{\mathrm{m}}<\varepsilon_{\mathrm{g}}$. The validity of Eq. (22) for low concentration and various frequencies, and the validity of Eq. (16) for concentrations near the limit of solubility of F381 will be demonstrated in the two following sections.

\subsection{The frequency dependence on the surfactant density of the monolayer during oscillation}

In Fig. 7, typical data from SHG measurements in the oscillating bubble configuration at four frequencies and four phase angles $\psi$ are shown. A phase-shift of $\sim 180^{\circ}$ between the modulations of the SHG-signal and the bubble area is clearly distinguishable. By use of Eqs. (20) and (21), the normalized surfactant density of the monolayer, $\Delta \Gamma_{\mathrm{m}} / \Gamma_{\mathrm{m}}$, was calculated, giving a value of $0.17-0.22$ for the four frequen- 


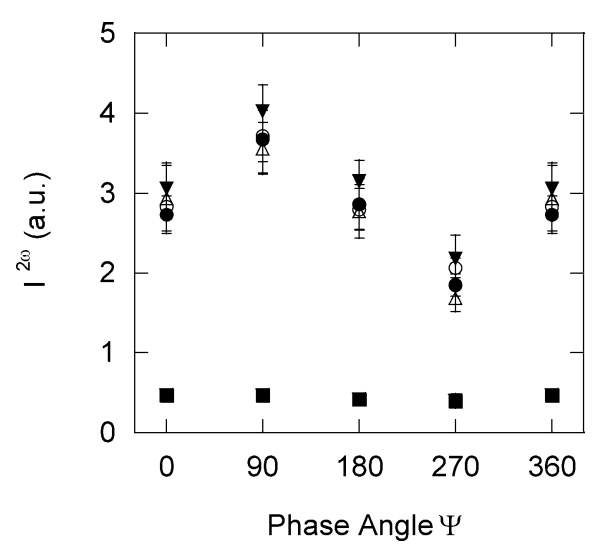

Fig. 7. SH intensity reflected by the oscillating bubble, as a function of phase angle $\psi$. Concentration: $30 \mu \mathrm{M}$. Oscillation frequency: $(\triangle) 10 \mathrm{~Hz}$, (Т) $20 \mathrm{~Hz},(\bigcirc) 40 \mathrm{~Hz},(\bullet) 60 \mathrm{~Hz}$; (ロ) water.

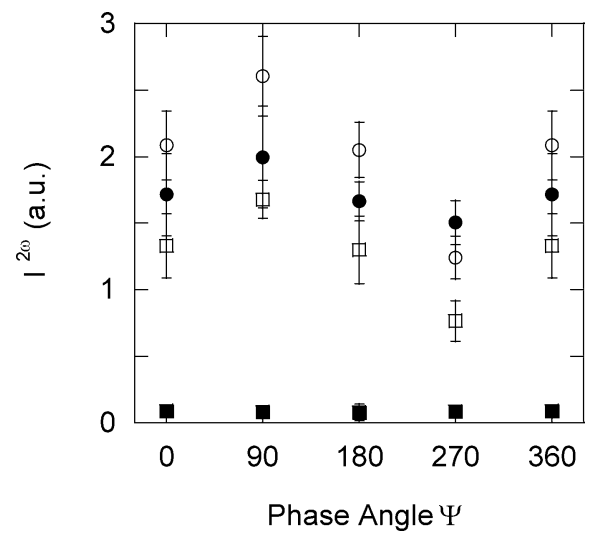

Fig. 8. SH intensity reflected by the oscillating bubble, as a function of bulk concentration of F381. Concentration: ( $\square) 10 \mu \mathrm{M},(\bigcirc) 30 \mu \mathrm{M},(\bullet) 150 \mu \mathrm{M}$. Bubble oscillation frequency: $40 \mathrm{~Hz}$; (ם) water.

cies shown in Fig. 7. The variation between the four frequencies is not significant, but is rather due to experimental inaccuracy, and changed slightly from one set of measurements to another. Consequently, the value of $\Delta \Gamma_{\mathrm{m}} / \Gamma_{\mathrm{m}}$ was, within experimental accuracy, independent of oscillation frequency in the range $10-60 \mathrm{~Hz}$. The same holds for all the concentrations studied here, i.e., $\Delta \Gamma_{\mathrm{m}} / \Gamma_{\mathrm{m}}$ was (within experimental accuracy) not frequency-dependent in the frequency range $10-60 \mathrm{~Hz}$ in the concentration regime $10-150 \mu \mathrm{M}$. This is in good agreement with the tensiometric studies in the oscillating bubble configuration, as well as with the assumption that the sublayer and the monolayer are in equilibrium during the whole deformation cycle in the studied frequency range.

\subsection{The bulk concentration dependence on surfactant density of the monolayer during oscillation}

Fig. 8 shows SHG measurements at four phase angles $\psi$ for three bulk concentrations of F381. The lowest bulk concentration $(10 \mu \mathrm{M})$ exhibits the lowest SHG signal, since a higher concentration increases the surface coverage and the $\mathrm{SH}$ signal generated at the tip of the bubble. The measured

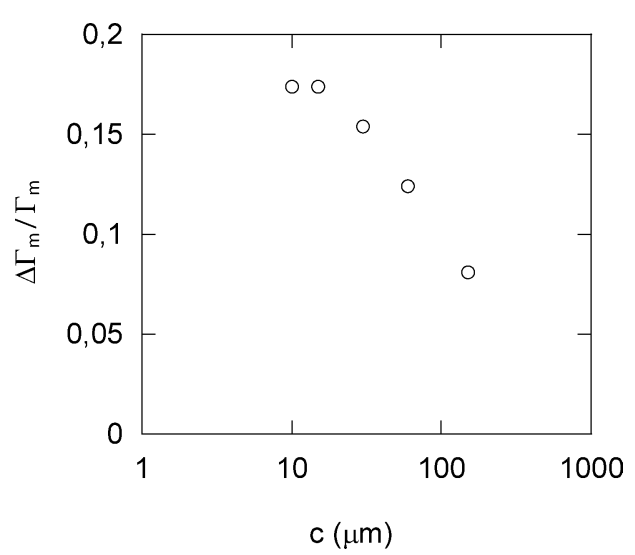

Fig. 9. Normalized surface density amplitude at the tip of the oscillating bubble, as measured by SHG.

SH signal is however smaller at $150 \mu \mathrm{M}$ than at $30 \mu \mathrm{M}$, as depicted in Fig. 8. The reason is that the absorption of $\mathrm{SH}$ light by the bulk solution increases with bulk concentration as well, and for the highest concentration $(150 \mu \mathrm{M})$ we estimate that $80-90 \%$ of the SH light is absorbed by the bulk solution. Since absorption plays such an important role at the higher concentrations, we choose to continue to look only at the normalized surfactant density of the monolayer amplitude $\left(\Delta \Gamma_{\mathrm{m}} / \Gamma_{\mathrm{m}}\right)$, which is not affected by bulk absorption.

From the data shown in Fig. 8, the normalized surfactant density of the monolayer as a function of concentration was calculated. The result is shown in Fig. 9 as a function of concentration. The three lowest concentrations exhibited in this set of measurements a $\Delta \Gamma_{\mathrm{m}} / \Gamma_{\mathrm{m}}$ ratio of $0.15-0.18$. Consequently, Eq. (22) is, within experimental accuracy, fulfilled for the three lowest concentrations. Taking into account that the phase angle $\varphi(f, c)$ of the measured modulus is approximately zero and the amount $E(f, c)$ is independent of frequency in the frequency range studied, we may conclude that for the concentrations 10,15 , and $30 \mu \mathrm{M}$, the dynamics of the system is described by the high frequency limit of the LvdT model. There, the exchange of the surfactant molecules between the monolayer and the sublayer can be neglected although the two phases are instantaneously in equilibrium due to the fast molecular exchange and the small values of $\omega_{\mathrm{m}}$. In the case of the higher concentration $60 \mu \mathrm{M}$, the ratio $\Delta \Gamma_{\mathrm{m}} / \Gamma_{\mathrm{m}}$ was in general smaller (Fig. 9), although the difference to $|\triangle A / A|$ is not far from the limit of the experimental accuracy. The deviation of the experimental result from Eq. (22) becomes however distinct at the highest concentration $(150 \mu \mathrm{M})$, where the ratio $\Delta \Gamma_{\mathrm{m}} / \Gamma_{\mathrm{m}}$ was $0.07 \pm 0.03$ (Figs. 8, 9). The vanishing phase angle $\varphi(f, c)$ of the measured surface dilatational modulus indicates that the subsurface and the monolayer are in equilibrium also at these concentrations, and the constant amount $E(f, c) \cong \varepsilon_{\mathrm{m}}$ hints to a very small molecular flux in the bulk $x<-d$. That means that $\Delta \Gamma / \Gamma=-\Delta A / A=\sim 0.18$. The decrease in the ratio $\Delta \Gamma_{\mathrm{m}} / \Gamma_{\mathrm{m}}$ suggests however an increase in the flow of molecules from the monolayer towards the sublayer in the surface compression mode, and a flow back to the monolayer 
in the surface expansion mode. That means, the surfactant molecules remain located in a small sublayer of thickness $d$ and the change in concentration in this layer has influence on the surface tension. Such behaviour was expected due to the dilatational modulus measurement and the explanation given by the modified model, but the first results of SHG experiments support this hypothesis strongly. It is only a consequence of the continuity equation and the physical explanation is not clear yet. Maybe, there is an energy and momentum transfer to the water molecules if the surfactant molecules push onto the surface.

\section{Conclusions}

The surface rheological properties of surfactant solutions are decisively influenced by molecular exchange processes at the surface. In the description of such processes, the models of fluid surfaces must be modified. This leads to a few new questions about the details of the structure of adsorption layers and their internal dynamics. Although molecular exchange rates and other parameters can be estimated by fitting of surface rheological measurements, details of dynamic surface models should be determined also by independent experiments. For this reason an experiment was realised that allows the monitoring of the adsorption state at an oscillating bubble using the surface SHG effect. In a first experimental series the setup was successfully tested with solutions of the fluoro surfactant F381. The measurements were done at frequencies where bulk-surface diffusion is widely suppressed, or negligible. The results show that in the low concentration range the relative change of the surfactant density of the monolayer, $\Delta \Gamma_{\mathrm{m}} / \Gamma_{\mathrm{m}}$, is equal to the negative value of the relative change in surface area of the bubble, whereas, near the limit of solubility, $\Delta \Gamma_{\mathrm{m}} / \Gamma_{\mathrm{m}}$ becomes smaller. This supports our hypothesis that at the higher concentrations, the molecules remain located in a sublayer between the bulk and the monolayer. This explains the frequently measured difference between the experimental high frequency limit of the surface elasticity, and the calculated Gibbs elasticity in the higher concentration range. Such new information is important for the introduction of detailed models describing molecular exchanges at a fluid surface, which are required to explain new results of surface rheological measurements. Already the first SHG measurements under dynamic condition improves the model.

\section{Acknowledgments}

The authors gratefully acknowledge financial support from the foundation Blanceflor Boncompagni-Ludovisi, born Bildt (J.Ö.), the Max-Planck-Gesellschaft, and the Deutsche Forschungsgemeinschaft. I. Bartsch is thanked for careful dynamic elasticity measurements and A. Kretzschmar and W. Stein for technical support.

\section{Appendix A. Nomenclature}

\begin{tabular}{|c|c|}
\hline$A$ & of bubble area during oscillation \\
\hline 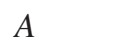 & bubble area \\
\hline$(x, t)$ & $\begin{array}{l}\text { surfactant concentration within the bulk }(x \leqslant 0 \text {, } \\
\text { molecules/unit volume) }\end{array}$ \\
\hline & bulk concentration far from the surface \\
\hline & $\begin{array}{l}\text { equilibrium sublayer concentration at } x=-d \text {, } \\
\text { standard assumption: } c_{\mathrm{s}}^{0}=c_{0}\end{array}$ \\
\hline & $\begin{array}{l}\text { dynamic sublayer concentration at } x=-d\left(c_{\mathrm{s}}=\right. \\
\left.c(-d, t)=c_{\mathrm{s}}^{0}+\Delta c_{\mathrm{s}}\right)\end{array}$ \\
\hline & bulk diffusion coefficient \\
\hline & sublayer thickness \\
\hline & magnitude of the surface dilational modulus \\
\hline & oscillation frequency of the bubble \\
\hline & limit frequency \\
\hline & incoming light intensity \\
\hline$I^{2 \omega}$ & outgoing second harmonic light intensity \\
\hline & wave number of the diffusion wave \\
\hline & $\begin{array}{l}\text { number of surfactant molecules within the surface } \\
\text { layer }\end{array}$ \\
\hline & surface dilatational modulus \\
\hline & $\begin{array}{l}\text { Gibbs elasticity resulting from the equilibrium } \\
\text { isotherm }\end{array}$ \\
\hline & modified Gibbs elasticity \\
\hline & experimental high frequency limit of the elasticity \\
\hline $3^{*}$ & phase shift describing kinetic exchange processes \\
\hline & $\begin{array}{l}\text { phase shift between bubble deformation and mea- } \\
\text { sured pressure modulation }\end{array}$ \\
\hline & $\begin{array}{l}\text { phase angle of the bubble during the detection of } \\
\text { the surface concentration }\end{array}$ \\
\hline & $\begin{array}{l}\text { surface concentration of the surfactant (molecules/ } \\
\text { unit area) }\end{array}$ \\
\hline 0 & surface concentration, static term \\
\hline$\Delta \Gamma$ & surface concentration, dynamic term \\
\hline$\Gamma_{\mathrm{m}}$ & surfactant concentration in monolayer \\
\hline$\Gamma^{*}$ & surfactant concentration in sublayer \\
\hline$\Gamma^{\prime}$ & fictive concentration \\
\hline$\Gamma_{\mathrm{w}}$ & surface concentration of solvent \\
\hline & compositional term of the surface tension \\
\hline $\mathrm{m}$ & $\begin{array}{l}\text { compositional term of the surface tension derived } \\
\text { from SHG measurements }\end{array}$ \\
\hline$\gamma^{\prime}$ & $\begin{array}{l}\text { complete surface tension including the influence of } \\
\text { dissipative processes }\end{array}$ \\
\hline & intrinsic surface dilatational viscosity \\
\hline & wavelength of diffusion wave \\
\hline $\begin{array}{l}\chi_{\text {eff }}^{(2)} \\
\omega=2 J\end{array}$ & $\begin{array}{l}\text { effective second-order susceptibility } \\
f \text { angular frequency }\end{array}$ \\
\hline$\omega_{\mathrm{g}}$ & $\begin{array}{l}\text { theoretical value of the molecular exchange para- } \\
\text { meter }\end{array}$ \\
\hline$g$ & modified molecular exchange parameter \\
\hline & $\begin{array}{l}\text { experimental value of the molecular exchange pa- } \\
\text { rameter }\end{array}$ \\
\hline
\end{tabular}




\section{References}

[1] B.P. Binks (Ed.), Modern Characterization Methods of Surfactant Systems, in: Surf. Sci. Ser., vol. 83, Dekker, New York, 1999.

[2] D. Möbius, R. Miller (Eds.), Novel Methods to Study Interfacial Layers, in: Stud. Interface Sci. Ser., vol. 11, Elsevier, Amsterdam, 2001.

[3] S.S. Dukhin, G. Kretzschmar, R. Miller, Dynamics of Adsorption at Liquid Interfaces, in: Stud. Interface Sci. Ser., vol. 1, Elsevier, Amsterdam, 1995.

[4] D. Möbius, R. Miller (Eds.), Drops and Bubbles in Interfacial Research, in: Stud. Interface Sci. Ser., vol. 6, Elsevier, Amsterdam, 1998.

[5] G. Kretzschmar, K. Lunkenheimer, Ber. Bunsen-Ges. Phys. Chem. 74 (1970) 107.

[6] K.-D. Wantke, R. Miller, K. Lunkenheimer, Z. Phys. Chem. (Leipzig) 261 (1980) 1177.

[7] K.-D. Wantke, K. Lunkenheimer, C. Hempt, J. Colloid Interface Sci. 159 (1993) 28.

[8] H. Fruhner, K.-D. Wantke, Colloids Surf. A 114 (1996) 53.

[9] K.-D. Wantke, H. Fruhner, J. Fang, K. Lunkenheimer, J. Colloid Interface Sci. 208 (1998) 34.

[10] H. Fruhner, K.-D. Wantke, K. Lunkenheimer, Colloids Surf. A 162 (1999) 193.

[11] K.-D. Wantke, H. Fruhner, J. Colloid Interface Sci. 237 (2001) 185.

[12] D.O. Johnson, K.J. Stebe, J. Colloid Interface Sci. 168 (1994) 21.
[13] D.O. Johnson, K.J. Stebe, J. Colloid Interface Sci. 182 (1996) 526.

[14] D.O. Johnson, K.J. Stebe, Colloids Surf. A 114 (1996) 41.

[15] J. Lucassen, R.S. Hansen, J. Colloid Interface Sci. 23 (1967) 319.

[16] J. Lucassen, M. van den Tempel, J. Colloid Interface Sci. 41 (1972) 491.

[17] J. Lucassen, M. van den Tempel, Chem. Eng. Sci. 27 (1972) 1283.

[18] J. Stauff, Kolloidchemie, Springer-Verlag, Berlin, 1960.

[19] A.W. Adamson, A.P. Gast, Physical Chemistry of Surfaces, Wiley, New York, 1997.

[20] Y.R. Shen, The Principles of Nonlinear Optics, Wiley, New York, 1984.

[21] K.B. Eisenthal, Chem. Rev. 96 (1996) 1343.

[22] G.L. Richmond, Chem. Rev. 102 (2002) 2693.

[23] S. Bae, M. Harke, A. Goebel, K. Lunkenheimer, H. Motschmann, D. Prescher, Langmuir 13 (1997) 6274.

[24] H. Motschmann, K. Lunkenheimer, J. Colloid Interface Sci. 248 (2002) 462.

[25] G.J. Simpson, K.L. Rowlen, J. Am. Chem. Soc. 121 (1999) 2635.

[26] J. Örtegren, K.-D. Wantke, H. Motschmann, Rev. Sci. Instrum. 74 (2003) 5167.

[27] V. Vogel, C. Mullin, Y.R. Shen, Langmuir 7 (1991) 1222.

[28] S. Lehmann, G. Busse, M. Kahlweit, R. Stolle, F. Simon, G. Marowsky, Langmuir 11 (1995) 1174.

[29] K. Lunkenheimer, J. Colloid Interface. Sci. 160 (1993) 509. 